\title{
Tracing Planets in Circumstellar Disks
}

\author{
Sebastian Wolf \\ Max Planck Institute for Astronomy, Königstuhl 17, 69117 Heidelberg, Germany \\ email: swolf@mpia.de
}

\begin{abstract}
We present simulations which demonstrate to which extent mid-infrared images and millimeter maps can be used to trace the location of giant planets in circumstellar disks. The most promising approach is to look for characteristic signatures in circumstellar disks caused by the interaction of giant planets with the disk. Numerical simulations show that these signatures are usually in size much larger than the planet itself and thus much easier to detect. The particular result of the planet-disk-interaction depends on the evolutionary stage of the disk. Primary signatures of planets embedded in disks are gaps in the case of young disks and characteristic asymmetric density patterns in debris disks. Radiative transfer simulations predict that high spatial resolution observations performed with instruments/telescopes that will become available in the near future will allow to trace the formation and evolution of planets in protoplanetary and debris disks.
\end{abstract}

Keywords. Protoplanetary disk, Planet formation, Planet-disk-interaction, Radiative transfer simulation, Multi-wavelength observation.

\section{Introduction}

Planets are expected to form in circumstellar disks, which are considered as the natural result of the protostellar evolution, at least in the case of low and intermediate mass stars. While a detailed picture of the evolution of the circumstellar environment, in particular of circumstellar disks, has been developed already, the planet formation process is in major parts still under discussion. There exist two major scenarios for planet formation. The first is characterized by three main phases: a) dust grain growth from submicron-sized particles to centimeter / decimeter-sized bodies via coagulation, followed by b) an agglomeration process that leads to the formation of (sub)kilometer-sized planetesimals, which c) form terrestrial (rocky) and Uranian (icy) planets or the cores of Jovian (gaseous) planets by further accretion of solid material (see, e.g., Pollack et al. 1996; Weidenschilling 1997). Alternatively, planetesimals may form via the gravitational instability of solids that have settled to the midplane of a circumstellar disk (Goldreich \& Ward 1973; Youdin \& Shu 2002). Adequate constraints from observations are required in order to either verify or to rule out existing hypotheses about these planet formation scenarios.

Once (proto-)planets have been formed, they may significantly alter the surface density profile of the disk and thus cause signatures in the disk that are much easier to find than the planets themselves. The appearance and type of these signatures depend on the mass and orbit of the planet, but even more on the evolutionary stage of the circumstellar disk. The most important of these signatures are gaps and spiral density waves in young circumstellar disks (see, e.g., Bryden et al. 1999; Kley 1999) and resonance structures in evolved systems, so-called debris disks (see, e.g., Liou \& Zook 1999). The importance of investigating these signatures lies in the possibility to use them for the search and the characterization of embedded planets. Therefore, disk features can provide constraints on the processes and timescales of planet formation. Indeed, several debris disks around 
nearby main-sequence stars show structures and asymmetries that are considered to result from planetary perturbations (Holland et al. 1998, 2003; Schneider et al. 1999; Koerner, Sargent, \& Ostroff 2001).

In this article we discuss selected features resulting from the planet-disk interaction which can be used to trace planets in circumstellar disks. We consider both, highresolution imaging techniques and spectroscopy as tools to constrain planetary parameters.

\section{Young Circumstellar Disks}

Hydrodynamic simulations of gaseous, viscous protoplanetary disks with an embedded protoplanet show that planets with masses $\gtrsim 0.1 \mathrm{M}_{\text {Jupiter }}$ produce significant perturbations in the disk's surface density (Bate et al. 2003). In particular, they may open and maintain a large gap. This gap, which is located along the orbit of the planet, may extend up to several astronomical units in width, depending on the mass of the planet and the hydrodynamic properties of the disk. Protoplanets also launch spiral waves. However, in the presence of turbulence, these waves appear to be diffused and dissipated (Nelson \& Papaloizou 2003). Furthermore, D'Angelo, Henning, \& Kley (2002) found smaller-scale spirals in the vicinity of the planet that are detached from the main ones: Along these small spirals the gas orbits the planet, representing a circumplanetary disk (see also Lubow, Seibert, \& Artymowicz 1999).

The observability of the outlined features has been investigated by Wolf et al. (2002). It was shown, that high-resolution images in the (sub)millimeter wavelength range as to be obtained with the Atacama Large Millimeter Array (ALMA) will allow to trace the gap, but not the large-scale spiral structure. Wolf \& D'Angelo (2005) performed more detailed simulations with the goal to answer the question whether the circumplanetary environment of a giant planet, embedded in a young circumstellar disk, can also be detected. The detection of a gap would already represent a strong indication of the existence of a planet, thus giving information such as planetary mass, viscosity, and pressure scale-height of the disk. However, the detection/non-detection of warm dust close to the planet would provide valuable additional constraints for the temperature and luminosity of the planet and on the density structure of the surrounding medium. It was shown that a detection of the circumplanetary accretion disk will indeed be possible (see Fig. 1). Two major constraints that limit the applicability of this approach to study planet formation were found: First, only for nearby objects at distances of typically less than $\sim 100 \mathrm{pc}$ the spatial resolution provided by $A L M A$ will be sufficiently high to allow to spatially separate the circumstellar disk and circumplanetary material from one another. Second, the emission from the circumplanetary environment is significantly strong only in the case of massive and thus young circumstellar disks. Furthermore, the signal of the radiation from the planet/circumplanetary material will be best distinguishable if a high planet-to-star mass ratio is targeted. We want to remark that in contrast to the most successful planet detection technique so far, based on radial velocity measurements (see, e.g., Mayor \& Queloz 1995, Marcy \& Butler 2000), the likelihood of detection does not increase with decreasing distance of the planet to the star (within the considered range of orbital radii of the planet of up to $5 \mathrm{AU}$ ).

The contribution of the planet to the net flux in the (sub)millimeter wavelength range by direct or scattered radiation and reemission of the heated dust in its vicinity - is negligible compared to the dust reemission from the entire disk. Furthermore, the planetary radiation significantly affects the dust reemission spectral energy distribution (SED) only in the near to mid-infrared wavelength range. However, since this spectral region is 

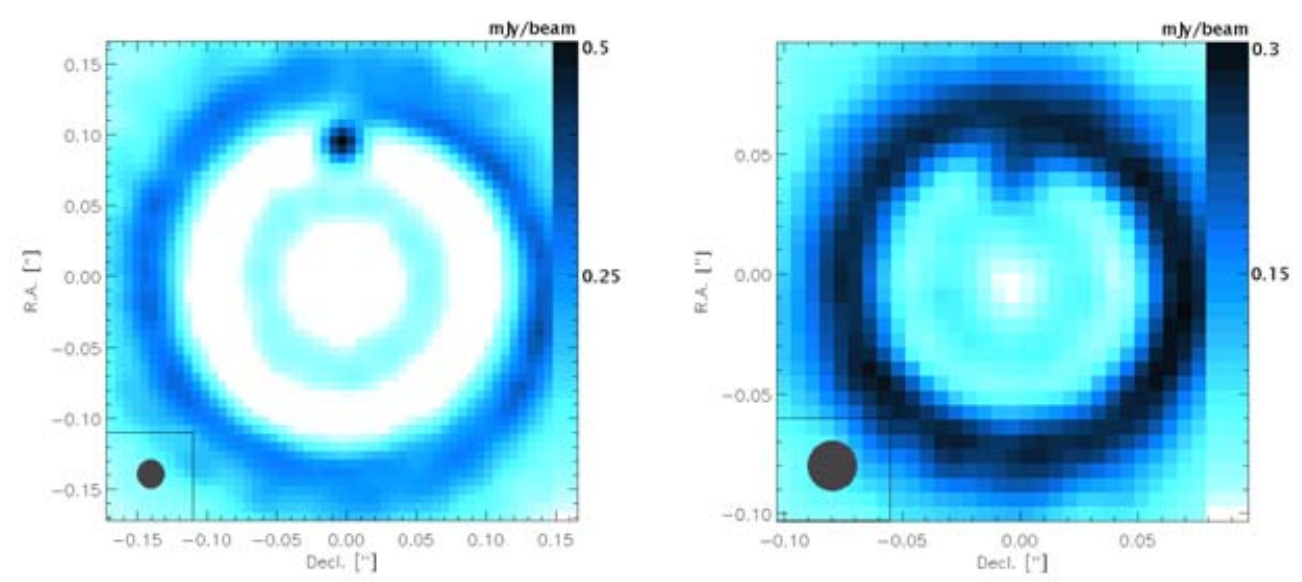

Figure 1. Simulation of $A L M A$ observations of a disk with an embedded $1 \mathrm{M}_{\text {jupiter }}$ planet around a $0.5 \mathrm{M}_{\text {sun }}$ star (orbital radius: $5 \mathrm{AU}$ ). The assumed distance is $50 \mathrm{pc}$ (left) $/ 100 \mathrm{pc}$ (right). The disk mass amounts to $M_{\text {disk }}=1.0 \times 10^{-2} \mathrm{M}_{\text {sun }}$ (total disk radius: $300 \mathrm{AU}$ ). Only structures above the $2 \sigma$-level are shown. The size of the combined beam is symbolized in the lower left edge of each image. Note the reproduced shape of the spiral wave near the planet and the slightly shadowed region behind the planet. [from Wolf \& D'Angelo 2005].

influenced also by the warm upper layers of the disk and the inner disk structure, the planetary contribution and thus the temperature/luminosity of the planet cannot be derived from the SED alone.

Since the location, depth, and width of a gap depend on the planetary mass and orbital parameters, these quantities can be constrained on the basis of high-resolution images. However, this interpretation will rely on the comparison with numerical simulations and thus on the physical processes considered. For example, the results from magnetohydrodynamic simulations of circumstellar disks with an embedded planet show, that the gaps are shallower and asymmetrically wider than in the case of purely hydrodynamic simulations, and the rate of gap-formation is slowed (Winter, Balbus, \& Hawley 2003).

\section{Debris Disks}

Debris disks are solar-system-sized dust disks with micron-sized grains produced as by-products of collisions between asteroid-like bodies left over from the planet formation process. In the case of our solar system, the debris of Jupiter-family short-period comets and colliding asteroids represents the dominant source of zodiacal dust located between Mars and Jupiter. A second belt of dust is located beyond the orbit of Neptune (see, e.g., Dermott et al. 1992; Liou, Dermott, \& Xu 1995). Besides the solar system, optical to mid-infrared images of $\beta$ Pic (see, e.g., Weinberger, Becklin, \& Zuckerman 2003) and submillimeter images of Vega, Fomalhaut, and $\epsilon$ Eri (Holland et al. 1998; Greaves et al. 1998) have revealed spatially resolved debris disks which were first inferred from observations of infrared flux excesses above photospheric values with IRAS. Since the mass of small grains in debris disks and therefore the thermal dust reemission from these disks is much smaller than in the case of T Tauri/HAe/Be circumstellar disks, only a very limited sample of observations exists so far.

The much lower optical depth and lower gas-to-dust mass ratio in debris disks (Zuckerman, Forveille, \& Kastner 1995; Dent et al. 1995; Liseau \& Artymowicz 1998; Greaves, Coulson, \& Holland 2000) let the stellar radiation - in addition to 

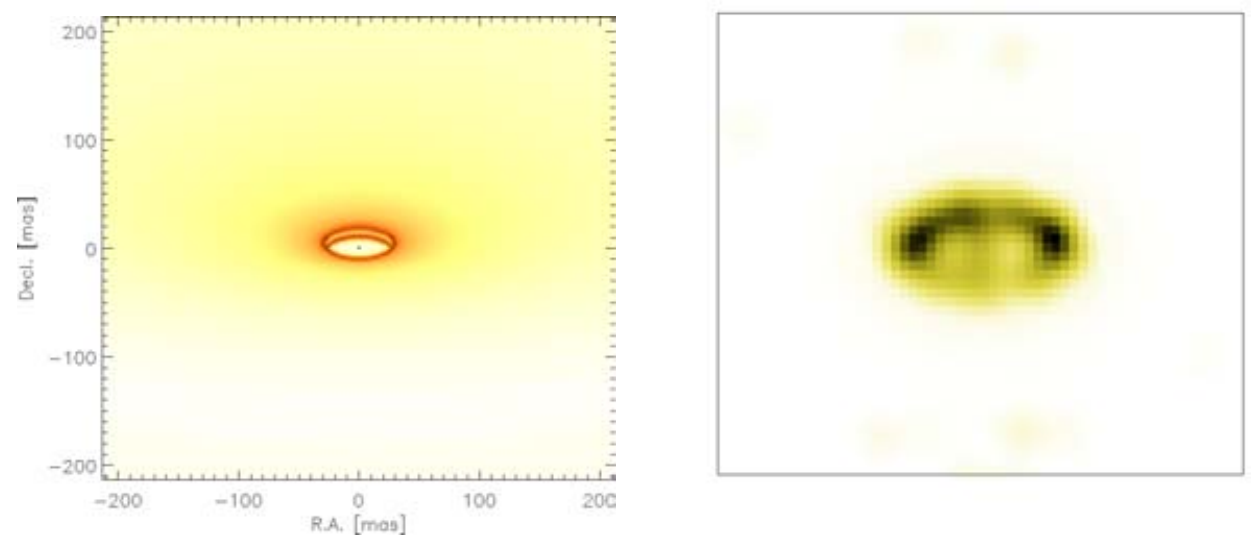

Figure 2. Simulated $10 \mu \mathrm{m}$ image of the inner region of a T Tauri circumstellar disk with a cleared inner region (radius: $4 \mathrm{AU}$ ), seen under an inclination of $60^{\circ}$ (assumed distance: $140 \mathrm{pc}$ ). Left: Original image; Right: Reconstructed image (not scaled), based on simulated VLTI observations combining 4 Telescope (APreS-MIDI/MATISSE concept study; see e.g., Lopez et al. 2005). [from Wolf et al. 2005].

gravitation - be responsible for the disk structure. The Poynting-Robertson effect, stellar wind drag, and - if existing - gravitational stirring by embedded planets are all important in determining the dust population and disk structure (see, e.g., Grady et al. 2000; Moro-Martín, Wolf, \& Malhotra 2005). Similar to T Tauri-like disks, however, embedded planets may alter the debris disk structure remarkably (see, e.g., Gor'kavyi et al. 1997; Kenyon \& Bromley 2001). In evolved, optically thin debris disks, a planet may produce an asymmetric resonant dust belt with one or more clumps and a central cavity void of dust via resonances and gravitational scattering (Ozernoy et al. 2000; Liou \& Zook 1999). The resulting characteristic density patterns are expected to provide strong indirect hints on the existence of planets in these disks. Kuchner \& Holman (2003) pointed out four basic high-contrast resonant structures a planet with eccentricity $\lesssim 0.6$ can create in a disk of dust released on low eccentric orbits: (i) a ring with a gap at the location of the planet, (ii) a smooth ring, (iii) a clumpy eccentric ring, and (iv) an offset ring plus a pair of clumps. The appearance/dominance of one of these structures mainly depends on the mass of the planet and the eccentricity of its orbit.

Observed SEDs of selected disks around T Tauri and debris-type disks have been found to show hints of inner cavities (void of small dust grains) such that the inner radius of the disk is apparently much larger than the sublimation radius of possible dust species. Examples among T Tauri disks are GM Aurigae (Koerner et al. 1993, Rice et al. 2003) and TW Hya (Calvet et al. 2002). In Fig. 2 the reconstructed image of a circumstellar disk with an inner hole is shown which could be obtained with the VLTI combining up to 4 Auxiliary Telescopes (Wolf et al. 2005). In several prominent debris disks, inner cavities have been found as well: $\beta$ Pic (inner radius: $20 \mathrm{AU}$ ), HR 4796 A (30-50 AU), $\epsilon$ Eri (50 AU), Vega (80 AU), and Fomalhaut (125 AU) - see, e.g., Dent et al. 2000; Wilner et al. 2002; Holland et al. 2003. Taking into account the physical processes responsible for the spatial dust density distribution in debris disks, scattering of particles by massive planets is assumed to be the major effect in explaining these large inner holes: Dust grains drifting inwards due to the Poynting-Robertson effect are likely to be scattered into larger orbits resulting in a lower dust number density within the planet's orbit. Clearing the inner disk results in a loss of hot dust which is mainly responsible for the near $/ \mathrm{mid}$ infrared shape of the SED (see Fig. 3). With increasing gap size the minimum value of 

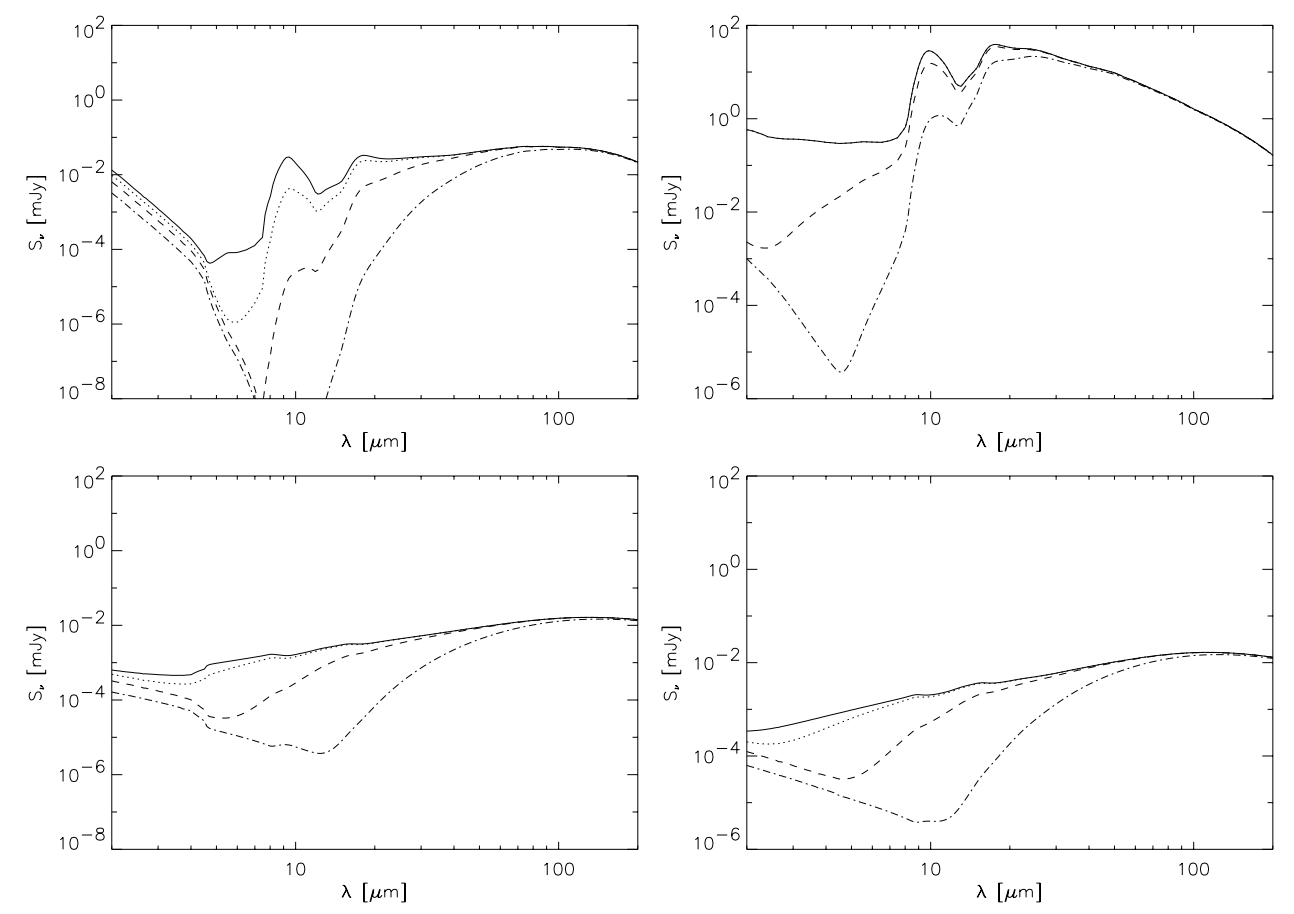

Figure 3. Influence of an inner gap on the SED of a debris disk. Inner disk radius: dust sublimation radius (solid line), 0.1 AU (dotted), $1 \mathrm{AU}$ (dashed), and $10 \mathrm{AU}$ (dash-dotted). Left/Right column: Fe-poor Silicate $\left(\mathrm{MgSiO}_{3}\right) /$ Fe-rich (Olivine, $\mathrm{MgFeSiO}_{4}$ ).

Top: $a=0.1 \mu \mathrm{m}$, Bottom: $a=1 \mathrm{~mm}$. Disk mass: $10^{-10} \mathrm{M}_{\text {sun }}$. [from Wolf $\mathscr{G}$ Hillenbrand 2003].

the mid-infrared flux becomes smaller and is shifted towards longer wavelengths. Detailed studies of the influence of planets on the SED of debris disks have been performed by Wolf \& Hillenbrand (2003) and Moro-Martín, Wolf, \& Malhotra (2005).

\section{Summary}

Numerical simulations convincingly demonstrate that high-resolution imaging performed with instruments/telescopes that will become available in the near future will allow detection of planets in circumstellar disks. However, scattering of stellar light and thermal emission by small grains (in the case of optically thick disks seen at high inclination also absorption) will make direct planet detection via imaging hardly feasible. But planets cause large-scale perturbations in the disks which will be observable with observatories like the Stratospheric Observatory For Infrared Astronomy (SOFIA), the James Webb Space Telescope (JWST), but also ground-based observatories/interferometers, such as $A L M A$ or the VLTI by spatially resolved mapping of the dust reemission. The particular type of perturbation depends on the evolutionary stage of the disk. Primary signatures of planets embedded in disks are gaps and circumplanetary accretion disks in the case of young disks and characteristic asymmetric density patterns in debris disks. Secondary signatures are, for instance, spiral structures in young disks or warps due to planets on inclined orbits. 


\section{Acknowledgements}

S. W. was supported by the German Research Foundation through the Emmy Noether grant WO $857 / 2-1$.

\section{References}

Bate, M.R., Lubow, S.H., Ogilvie, G.I., \& Miller, K.A. 2003, MNRAS 341, 213

Bryden, G., Chen, X., Lin, D.N.C., Nelson, R.P., \& Papaloizou, J.C.B. 1999, ApJ 514, 344

Calvet, N., D'Alessio, P., Hartmann, L., Wilner, D., Walsh, A., \& Sitko, M. 2002, ApJ 568, 1008

Dent, W.R.F., Greaves, J.S., Mannings, V., Coulson, I.M., \& Walther, D.M. 1995, MNRAS 277, L25

Dent, W.R.F., Walker, H.J., Holland, W.S., \& Greaves, J.S. 2000, MNRAS 314, 702

Dermott, S.F., Durda, D.D., Gustafson, B.A., Jayaraman, S., Xu, et al. 1992, in Asteroids, Comets, Meteors A.W. Harris \& E. Bowell (eds.), 153

D'Angelo, G., Kley, W., \& Henning, Th. 2003, ApJ 586, 540

Goldreich, P., \& Ward W.R. 1973, ApJ 183, 1051

Gor'kavyi, N.N., Ozernoy, L.M., Mather, J.C., \& Taidakova, T. 1997, ApJ 488, 268

Grady, C.A., Sitko, M.L., Russell, R.W., Lynch, D.K., Hanner, M.S., et al. 2000, in Protostars and Planets IV V. Mannings, A.P. Boss, S.S. Russell (eds.), University of Arizona Press, p. 613

Greaves, J.S., Holland, W.S., Moriarty-Schieven, G., et al. 1998 ApJ 506, L133

Greaves, J.S., Coulson, I.M., \& Holland, W.S. 2000, MNRAS 312, L1

Holland, W.S., Greaves, J.S., Dent, W.R.F., Wyatt, M.C., Zuckerman, B., et al. 2003, ApJ 582, 1141

Holland, W.S., Greaves, J.S., Zuckerman, B., et al. 1998, Nature 392, 788

Kenyon, S.J., \& Bromley, B.C. 2001, ApJ 121, 538

Kley, W. 1999, MNRAS 303, 696

Kley, W. 2000, MNRAS 313, L47

Koerner, D.W., Sargent, A.I., \& Ostroff, N. 2001, ApJ 560, L181

Kuchner, M.J., \& Holman, M.J. 2003, ApJ 588, 1110

Liou, J.-C., Dermott, S.F., \& Xu, Y.-L. 1995, Planet. Space Sci. 43, 717

Liou, J.-C., \& Zook, H.A. 1999, AJ 118, 580

Liseau, R., \& Artymowicz, P. 1998, A\&A 334, 935

Lopez, B., Wolf, S., Dugue, M., Graser, U., Mathias, Ph., et al. 2005, in Proc. of "The Power of Optical/Infrared Interferometry", ESO Garching (Germany)

Lubow, S. H., Seibert, M., \& Artymowicz, P. 1999, ApJ 526, 1001

Marcy, G.W., \& Butler, R.P. 2000, PASP 112, 137

Mayor, M., \& Queloz, D. 1995, Nature 378, 355

Moro-Martín, A., Wolf, S., \& Malhotra, R. 2005, ApJ 129, 1049

Nelson, R.P., \& Papaloizou, J.C.B. 2003, MNRAS 339, 993

Ozernoy, L.M., Gorkavyi, N.N., Mather, J.C., \& Taidakova, T.A. 2000, ApJ, 537, L147

Pollack, J.B., Hubickyj, O., Bodenheimer, P., Lissauer, J.J., Podolak, M., \& Greenzweig, Y. 1996, Icarus 124, 62

Schneider, G., Smith, B.A., \& Becklin, E.E. 1999, ApJ 513, L127

Weinberger, A.J., Becklin, E.E., \& Zuckerman, B. 2003, ApJ 584, L33

Wilner, D.J., Holman, M.J., Kuchner, M.J., \& Ho, P.T.P. 2002, ApJ 569, L115

Winters, W.F., Balbus, S.A., \& Hawley, J.F. 2003, ApJ 589, 543

Wolf, S., \& D'Angelo, G. 2005, ApJ 619, 1114

Wolf, S., Gueth, F., Henning, Th., \& Kley, W. 2002, ApJ 566, L97

Wolf, S., \& Hillenbrand, L.A. 2003, ApJ 569, 603

Wolf, S., Lopez, B., Augereau, J.-Ch., Berruyer, N., Chesneau, O., et al. 2005, "Mid Infrared VLTI Imager - APreS-MIDI/MATISSE Science Case Study", subm. to ESO

Youdin, A.N., \& Shu, F.H. 2002, ApJ 580, 494

Zuckerman, B., Forveille, T., \& Kastner, J.H. 1995, Nature 373, 494 